\title{
Long Term Outcome of Bisphosphonate Therapy in Patients with Primary Hyperparathyroidism
}

\author{
Dalitso Segula*, Tanya Nikolova, Eileen Marks, Lakshminarayan Ranganath, \\ Vinita Mishra \\ Department of Clinical Biochemistry \& Metabolic Medicine, Royal Liverpool \& Broadgreen University Hospital \\ NHS Trust, Liverpool, UK \\ Email: ${ }^{*}$ dalitsosegula@gmail.com
}

Received 25 May 2014; revised 24 June 2014; accepted 16 July 2014

Copyright (C) 2014 by authors and Scientific Research Publishing Inc.

This work is licensed under the Creative Commons Attribution International License (CC BY). http://creativecommons.org/licenses/by/4.0/

c) (7) Open Access

\begin{abstract}
Context: Primary hyperparathyroidism (PHPT) is commonly associated with reduced bone mineral density (BMD) presenting with osteoporosis, increasing the risk of bone fragility fractures in these patients. Bisphosphonates, due to their anti-resorptive action, are known to improve the BMD and reduce the risk of bone fragility fractures. Therefore, bisphosphonates are considered as an alternative to surgical treatment in managing osteoporosis in PHPT patients. Aim: The aim of this observational study was to assess the effect of long term bisphosphonate therapy on BMD, bone fragility fracture and biochemical markers of bone metabolism in patients with PHPT. Methodology: Fifty patients (mean age 74 years) with PHPT who were treated with long term bisphosphonate therapy were studied retrospectively. The mean baseline (before commencing bisphosphonate therapy) BMD T-scores for lumbar spine (L2-L4) and left femoral neck were $\mathbf{- 2 . 5}$ and -2.1 , respectively. Fourteen patients had bone fragility fractures before initiation of bisphosphonate therapy. Results: After an average of 5 years of bisphosphonate treatment, there was a significant increase in lumbar BMD T-score $(-2.5$ to $-2.1, p=0.013)$ and a non-significant change in left femoral neck BMD T-score $(-2.1$ to $-2.2, p=0.497)$. There was no increase in bone fragility fracture rate $(p=0.167)$. Serum corrected calcium reduced from $2.74 \mathrm{mmol} / \mathrm{L}$ to $2.60 \mathrm{mmol} / \mathrm{L}(p<$ $0.001)$ and urine calcium to creatinine ratio from 0.70 to $0.55(p<0.0001)$, both within the reference range. Conclusions: Our study suggests that long term bisphosphonate therapy improves lumbar BMD and prevents increase in bone fragility fracture rate. Additionally it improves hypercalcaemia in PHPT.
\end{abstract}

\section{Keywords}

Primary Hyperparathyroidism, Bisphosphonates, Hypercalcaemia, Bone Mineral Density,

${ }^{*}$ Corresponding author.

How to cite this paper: Segula, D., Nikolova, T., Marks, E., Ranganath, L. and Mishra, V. (2014) Long Term Outcome of Bisphosphonate Therapy in Patients with Primary Hyperparathyroidism. International Journal of Clinical Medicine, 5, 829-835. http://dx.doi.org/10.4236/ijcm.2014.514111 


\section{Bone Fragility Fractures}

\section{Introduction}

Primary hyperparathyroidism (PHPT) is a common cause of hypercalcaemia among outpatients [1]. It is estimated that $0.3 \%$ of the general population have PHPT and $1 \%-3 \%$ of postmenopausal women suffer from it [2]. Surgical treatment is clearly indicated in PHPT patients who present with severe hypercalcaemia, renal stone, osteoporosis and reduced renal function [3]. Nonetheless, studies have reported that $80 \%$ of patients who present with PHPT are asymptomatic (serum calcium $<3 \mathrm{mmol} / \mathrm{L}$ and no renal stones) [4]. It has been suggested that conservative approach may be more reasonable than surgical intervention in the management of stable asymptomatic PHPT [5].

Bone remodelling involves the removal of mineralized bone by osteoclasts (bone resorption) followed by formation of bone matrix by osteoblasts (bone formation) that is subsequently mineralised. Osteoporosis is characterised by an imbalance between osteoclast and osteoblast activities (bone uncoupling), resulting in impaired bone remodelling. Raised parathyroid hormone (PTH) in PHPT increases bone loss by stimulating the osteoclastic bone resorption [6]. Increased rate of bone turnover is usually determined by measuring the serum concentration of C-terminal telopeptide (CTx), which is a cross-link peptide sequence of type I bone collagen. Biochemically, raised PTH, due to its direct effect on bone, increases not only CTx but also calcium both in blood and urine. Clinically, this results in reduced BMD, osteoporosis, impairment in renal functions and renal stones.

Studies have shown that anti-resorptive treatment in the form of bisphosphonates is as effective as surgical treatment in improving BMD in patients with PHPT [7]. Bisphosphonates are analogues of inorganic pyrophosphates. Bisphosphonates attach to hydroxyapatite binding sites on bony surfaces, especially surfaces undergoing active bone resorption. When osteoclasts begin to resorb bone that is impregnated with bisphosphonate, the bisphosphonate released during resorption impairs the ability of the osteoclasts to form the ruffled border, to adhere to the bony surface, and to produce the protons necessary for continued bone resorption. In addition to this, bisphosphonates causes apoptosis of osteoclasts by inhibiting farnesyldiphosphate synthase in osteoclast cells, resulting in disruption of a number of pathways including cytoskeletal organisation, cell proliferation, and survival [8] and thus reduces osteoclast activity. Bone formation is often reduced by bisphosphonates, which is probably an indirect effect of inhibition of bone resorption. Bisphosphonates that contain nitrogen (such as alendronate, risedronate, ibandronate, and zoledronic acid) have the most potent antiresorptive properties and are the most commonly used drugs in the treatment of osteoporosis. Large randomized controlled trials in post menopausal osteoporosis women have shown significant reduction in vertebral, non-vertebral and hip fractures [9] with bisphosphonates. Both the National Institute for Health and Clinical Excellence (NICE) UK and the UK National Osteoporosis Guideline Group recommend bisphosphonate as the first line treatment option for primary or secondary prevention of fracture in postmenopausal women [10] [11].

The objective of this retrospective observational study was to primarily assess the effect of long-term bisphosphonate therapy on BMD in patients with PHPT. Besides this, we also examined the effect of long-term bisphosphonate therapy on bone fragility fractures and biochemical markers of bone turnover in PHPT.

\section{Methodology}

\subsection{Study Design}

This was a retrospective observational study carried out in patients with PHPT who attended the bone metabolic clinic for management of osteoporosis. Demographic and clinical data were collected from patients' charts.

\subsection{Study Population}

Fifty patients with PHPT treated with bisphosphonate therapy over an average of $5 \pm 3$ years (mean \pm SD) were studied retrospectively. The mean age was $74 \pm 9$ years (mean \pm SD), 47 patients were females. All the study patients presented with reduced BMD (Table 1) before starting the bisphosphonate therapy (baseline). The 
Table 1. Bone mineral status at baseline $(\mathrm{N}=50)$.

\begin{tabular}{cc}
\hline Osteoporosis, $n(\%)$ & $30(60)$ \\
Osteoporosis and fragility fracture, $n(\%)$ & $12(24)$ \\
Osteopenia, $n(\%)$ & $6(12)$ \\
Osteopenia and fragility fracture, $n(\%)$ & $2(4)$ \\
\hline
\end{tabular}

average baseline $T$-scores for lumbar spine (L2-L4) and left femoral neck were -2.5 and -2.1 , respectively. Patients were commenced on either intravenous or oral bisphosphonate therapy. Oral bisphosphonates can cause gastrointestinal adverse effects including oesophagitis, gastritis, dyspepsia, oesophageal reflux, nausea, abdominal pain, and diarrhoea. All our study patients were commenced on oral bisphosphonates but $36 \%$ were changed to intravenous bisphosphonate therapy as they presented with gastrointestinal symptoms with oral bisphosphonate therapy. Besides gastrointestinal problems observed with bisphosphonates there are long term adverse effects such as osteonecrosis of jaw and atypical fractures [12] [13] which have been reported with bisphosphonate treatment. None of our study patients presented with these problems during the period they were having bisphosphonate therapy.

\subsection{Biochemistry Measurements}

The laboratory results were collected from a laboratory database (Institute of Chemical Education Laboratory Database). Biochemistry data were collected at the baseline and after completion of bisphosphonate therapy.

\subsection{Data Analysis}

Mean or median values at baseline (at start of bisphosphonate treatment) were compared with values after treatment. The paired t-test was used to compare mean values of corrected calcium, serum creatinine, urine calcium to creatinine ratio, serum CTx and PTH. The Related Samples Wilcoxon Signed Rank test was used to compare median values of $T$-scores and McNemars test was used to assess if there was a significant difference in fragility fracture rate after treatment. A $p<0.05$ defined statistical significance. SPSS version 21 statistical software packages were used for the analysis of the data.

\section{Results}

\subsection{Primary Outcome of Bisphosphonate Therapy}

\section{Effect on BMD}

After an average of 5 years of bisphosphonate treatment, lumbar spine (L2-L4) BMD increased significantly to $-2.1(p=0.013)$ and there was a non-significant increase of BMD in the left femoral neck (Table 2).

\subsection{Secondary Outcome of Bisphosphonate Therapy}

\subsubsection{Effect on Bone Fragility Fracture}

Fourteen out of the 50 patients had bone fragility fractures at baseline (Table 3). During therapy one patient had a recurrent fracture whereas six patients who did not have fractures at baseline, had bone fragility fractures $(n=$ 7). There was no significant change in the rate of bone fragility fracture $(p=0.167)$ following bisphosphonate therapy.

\subsubsection{Effect on Biochemical Markers of PHPT}

There was a significant decrease in serum corrected calcium from $2.74 \mathrm{mmol} / \mathrm{L}$ to $2.60 \mathrm{mmol} / \mathrm{L}(p<0.001)$ and urine calcium to creatinine ratio from 0.70 to $0.55(p<0.0001)$. No significant change was observed in PTH, renal functions and CTx (Table 4).

\section{Discussion}

We observed a significant increase in lumbar spine BMD in patients with PHPT, who received bisphosphonate 
Table 2. Comparison of lumbar spine and femoral neck mineral bone density before and after bisphosphonate treatment.

\begin{tabular}{cccc}
\hline Bone mineral density & Baseline T-score & Post-treatment T-score & $p^{\text {-value }}$ \\
\hline $\begin{array}{c}\text { Lumbar spine, L2-L4 } \\
\text { median (IQR) }\end{array}$ & $-2.5(-3.1,-1.4)$ & $-2.1(-2.9,-0.9)$ & 0.013 \\
$\begin{array}{c}\text { Left femoral neck } \\
\text { median (IQR) }\end{array}$ & $-2.1(-2.6,-1.6)$ & $-2.2(-2.5,-1.7)$ & 0.497 \\
\hline
\end{tabular}

IQR: Interquantile range, ${ }^{*} p$ value $<0.05$ significant.

Table 3. Bone fragility fractures at baseline and during bisphosphonate treatment.

\begin{tabular}{|c|c|c|c|}
\hline Patient & Baseline fracture & $\begin{array}{l}\text { During-treatment fracture (number of years of } \\
\text { bisphosphonate treatment before fracture) }\end{array}$ & $\begin{array}{l}\text { Total duration of } \\
\text { treatment in years }\end{array}$ \\
\hline 1 & $\begin{array}{c}\text { Vertebra } \\
\text { Wrist }\end{array}$ & - & 5 \\
\hline 2 & $\begin{array}{c}\text { Vertebra } \\
\text { Hip }\end{array}$ & - & 6 \\
\hline 3 & Vertebra & - & 10 \\
\hline 4 & Hip & - & 6 \\
\hline 5 & $\begin{array}{c}\text { Shoulder } \\
\text { Radius }\end{array}$ & - & 7 \\
\hline 6 & $\begin{array}{c}\text { Ulnar } \\
\text { Humerus }\end{array}$ & - & 6 \\
\hline 7 & Vertebra & - & 8 \\
\hline 8 & Hip & - & 4 \\
\hline 9 & Vertebra & - & 4 \\
\hline 10 & Shoulder & - & 1 \\
\hline 11 & Elbow & - & 1 \\
\hline 12 & Vertebra & - & 2 \\
\hline 13 & $\begin{array}{c}\text { Vertebra } \\
\text { Wrist }\end{array}$ & - & 2 \\
\hline 14 & Vertebra & - & - \\
\hline 15 & - & Wrist (6) & 7 \\
\hline 16 & - & Rib (11) & 11 \\
\hline 17 & - & Rib (3) & 8 \\
\hline 18 & - & $\begin{array}{c}\text { Vertebra (7) } \\
\text { Hip (10) }\end{array}$ & 10 \\
\hline 19 & - & $\begin{array}{c}\text { Humerus (5) } \\
\text { Wrist (6) } \\
\text { Shoulder (6) } \\
\text { Elbow (6) }\end{array}$ & 6 \\
\hline 20 & - & Vertebra (3) & 3 \\
\hline
\end{tabular}


Table 4. Comparison of bone markers and other biochemical parameters before and after bisphosphonate treatment.

\begin{tabular}{cccc}
\hline Analytes (reference range) & Baseline mean value (SD) & Post-treatment mean value (SD) & $p$-value \\
\hline $\begin{array}{c}\text { Serum adjusted calcium } \\
\text { mmol/L }(2.20-2.60)\end{array}$ & $2.74(0.13)$ & $2.60(0.18)$ & $<0.0001$ \\
$\begin{array}{c}\text { Urine calcium: creatinine } \\
\text { ratio }(0.3-0.7)\end{array}$ & $0.70(0.22)$ & $0.55(0.25)$ & $<0.0001$ \\
$\begin{array}{c}\text { Serum creatinine } \\
\mu \text { mol/L }(60-110)\end{array}$ & $99.5(26.9)$ & $96.4(31.7)$ & 0.930 \\
$\begin{array}{c}\text { Serum PTH } \\
\text { pmol/L }(1.2-5.8)\end{array}$ & $10.8(5.7)$ & $10.3(5.1)$ & 0.668 \\
$\begin{array}{c}\text { Serum CTx, } \\
\mu \mathrm{g} / \mathrm{L}(0.1-0.5)\end{array}$ & $0.30(0.14-0.45)$ & $0.26(0.14-0.39)$ & 0.148 \\
\hline
\end{tabular}

PTH: parathyroid hormone; CTx: C-terminal telopeptide; SD: standard deviation.

therapy over an average period of 5 years. Anti-resorptive treatments such as bisphosphonates have shown a comparable increase in BMD as observed after surgical intervention in PHPT or in eucalcaemic population [7]. Since PHPT confers an increased risk of bone loss, bisphosphonates may be a reasonable alternative to surgery in patients with PHPT. PTH is preferentially known to affect the cortical bone more compared to trabecular bone [14]. Paradoxically, our study patients had lower bone density at lumbar spine (trabecular bone) compared to left femoral neck (cortical bone) before they were commenced on bisphosphonate therapy. Studies have shown that this effect of PTH may be due to its long-term biphasic action, which causes bone loss throughout the skeleton [7]. Bisphosphonates, by inhibiting the bone resorption, increase BMD in PHPT patients. We observed this affect in our study patients who received bisphosphonates for an average period of 5 years. Bisphosphonates are known to increase BMD significantly both at lumbar spine and left femoral neck density [15] [16] in PHPT. Nevertheless, we observed a significant increase in lumbar spine density and no significant change in left femoral neck density in our PHPT patients. The reason for this observed effect may be that studies so far have reported the short-term effect of bisphosphonates (2 years) on BMD in PHPT [7] [16] where as we studied the long-term effect of bisphosphonates on BMD. Besides bisphosphonates, hormone replacement therapies such as estrogens have also been reported to increase BMD in PHPT [17] [18].

The fracture risk in PHPT increases by 1.5 to 2 fold compared to eucalcaemic population [19]. The PHPT patients in our study presented with fractures involving both trabecular and cortical bone (Table 3). This is contrary to the action of PTH on skeleton, which predominantly results in greater cortical bone loss [14]. However there is no clear evidence that in PHPT, fracture risk will be higher in skeleton predominantly rich in cortical bone [19]. We did not observe any significant increase in fracture rate in our cohort of study patients after receiving bisphosphonate therapy over an average period of 5 years. This was reflected by a non-significant change in bone resorption marker CTx at the end of treatment. Bisphosphonates are well known to reduce the fracture risk in eucalcaemic osteoporosis patients [20]. Thus, it has been presumed that bisphosphonates will have a similar action on bone fragility fractures in PHPT patients. Before starting bisphosphonate treatment 14 PHPT patients (28\%) in our study had bone fragility fracture (baseline). In this cohort, only one patient had a recurrent fracture after 4 years of bisphosphonate treatment. Thus, bisphosphonates were able to prevent recurrent bone fragility fractures in all 14 patients except one, which corresponded with the significant increase in BMD. During the bisphosphonate therapy, 6 PHPT patients had new bone fragility fractures after an average period of 6 years of post therapy (Table 3). In total, there were 7 patients (including one who had recurrent fracture) patients (14\%) who had fragility fractures when they were on bisphosphonate treatment. The mean serum 25(OH) vitamin D levels in these seven patients was $27.9 \mathrm{nmol} / \mathrm{L}(25(\mathrm{OH})$ vitamin $\mathrm{D}$ below $30 \mathrm{nmol} / \mathrm{L}$ suggest deficiency), suggesting the association of low vitamin D status and bone fragility fracture [21]. Therefore, we presume that vitamin D deficiency may have contributed to the bone fragility fractures in our PHPT patients in spite of being on bisphosphonate treatment. Recent meta-analysis suggests that optimal vitamin D levels $(25(\mathrm{OH})$ vitamin D above $60 \mathrm{nmol} / \mathrm{L}$ ) are most beneficial in reducing the risk of hip and non-vertebral fractures in eucalcaemic elderly population [22]. There is no evidence that correcting low vitamin D status will reduce the bone 
fragility fractures in PHPT patients. Nevertheless, observational studies show an inverse association of size of parathyroid adenoma, bone turnover, levels of serum calcium, PTH and trabecular BMD with 25(OH) vitamin D [23] [24]. Thus it has been recommended to correct the low vitamin D status and maintaining 25(OH)D above $50 \mathrm{nmol} / \mathrm{L}$ in PHPT [5]. Although there was a reduction in fracture rate in our study patients, this failed to achieve significance presumably due to a small sample size. None of the previous studies have reported the effect of anti-resorptive treatment on fracture risk in PHPT patients.

We observed a significant reduction in calcium both in blood and urine following bisphosphonate therapy suggesting that bisphosphonates are able to ameliorate the effect of raised PTH on bone resorption. Previous studies have shown a similar finding [25] [26] emphasising the use of bisphosphonates in the management of hypercalcaemia in PHPT.

Additionally, there was no deterioration in renal function with bisphosphonate therapy in our study patients (Table 4). Usually, hypercalcaemia in PHPT results into deterioration in renal functions as it induces nephrogenic diabetes inspidus [27]. Since there was a significant improvement in serum corrected calcium levels following bisphosphonate therapy in our cohort, we did not observe deterioration in renal function. At the same time, several studies have reported no decline in renal functions in PHPT [28].

\section{Conclusion}

Our study suggests that long-term bisphosphonate therapy in PHPT improves lumbar BMD, prevents increase in rate of bone fragility fractures and improves hypercalcaemia. We recommend consideration of long-term bisphosphonate therapy in patients with PHPT. Randomized controlled trials are required to ascertain effect of vitamin D supplementation on fragility fracture rate in PHPT.

\section{Study Limitations}

Our study does have some limitations. Firstly it is an observational retrospective study and cause-effect relationship cannot be ascertained in this design. However, the findings of our study do concur with previous randomised control trials, which have shown increase in BMD with bisphosphonates. Secondly, it is likely that differences were not observed in some variables due to the size of the cohort. The sample size calculation was not indicated as we had included all patients with PHPT who had their records available.

Further prospective randomised controlled trials are required to assess the effect of bisphosphonate therapy and vitamin D supplementation on bone fragility fractures in PHPT patients.

\section{References}

[1] Tierney, L.M., McPhee, S.J. and Papadakis, M.A. (2006) Current Medical Diagnosis and Treatment. McGraw-Hill Professional, 901.

[2] Melton Jr., L.J. (2002) The Epidemiology of Primary Hyperparathyroidism in North America. Journal of Bone and Mineral Research, 17, N12-N17.

[3] Bilezikian, J.P., Khan, A.A. and Potts, J.T.J. (2009) Guidelines for the Management of Asymptomatic Primary Hyperparathyroidism: Summary Statement from the Third International Workshop. Journal of Clinical Endocrinology and Metabolism, 94, 335-339. http://dx.doi.org/10.1210/jc.2008-1763

[4] Fraser, W.D. (2009) Hyperparathyroidism. Lancet, 374, 145-158. http://dx.doi.org/10.1016/S0140-6736(09)60507-9

[5] Gray, A. (2012) Nonsurgical Management of Mild Primary Hyperparathyroidism-A Reasonable Option. Clinical Endocrinology, 77, 639-644.

[6] Melton, L.J., Khosla, S., Atkinson, E.J., et al. (1997) Relationship of Bone Turnover to Bone Density and Fractures. Journal of Bone and Mineral Research, 12, 1083-1091. http://dx.doi.org/10.1359/jbmr.1997.12.7.1083

[7] Sankaran, S., Gamble, G., Bolland, M., et al. (2010) Skeletal Effects of Interventions in Mild Primary Hyperparathyroidism: A Meta-Analysis. Journal of Clinical Endocrinology and Metabolism, 95, 1653-1662. http://dx.doi.org/10.1210/jc.2009-2384

[8] Rodan, G.A. and Fleisch, H.A. (1996) Bisphosphonates: Mechanism of Action. Journal of Clinical Investigation, 97, 2692-2696. http://dx.doi.org/10.1172/JCI118722

[9] Black, D., Cummings, S., Karpf, D., Cauley, J.A., Thompson, D.E., Nevitt, M.C., et al. (1996) Randomised Trial of Effect of Alendronate on Risk of Fracture in Women with Existing Vertebral Fractures. Lancet, 348, 1535-1541. http://dx.doi.org/10.1016/S0140-6736(96)07088-2 
[10] National Institute for Health and Clinical Excellence (2011) Osteoporosis-Secondary Prevention including Strontium Ranelate. Alendronate, Etidronate, Risedronate, Raloxifene, Strontium Ranelate and Teriparatide for the Secondary Prevention of Osteoporotic Fragility Fractures in Postmenopausal Women. Technical Appraisal 161. http://www.nice.org.uk/TA16

[11] Compston, J., Cooper, A., Cooper, C., Francis, R., Kanis, J.A., Marsh, D., et al. (2009) Guidelines for the Diagnosis and Management of Osteoporosis in Postmenopausal Women and Men from the Age of 50 Years in the UK. Maturitas, 62, 105-108. http://dx.doi.org/10.1016/j.maturitas.2008.11.022

[12] Khosla, S., Burr, D., Cauley, J., Dempster, D.W., Ebeling, P.R., Felsenberg, D., et al. (2007). Bisphosphonate-Associated Osteonecrosis of the Jaw: Report of a Task Force of the American Society for Bone and Mineral Research. Journal of Bone and Mineral Research, 22, 1479-1491. http://dx.doi.org/10.1359/jbmr.0707onj

[13] Compston, J. (2011) Pathophysiology of Atypical Femoral Fractures and Osteonecrosis of the Jaw. Osteoporosis International, 22, 2951-2961. http://dx.doi.org/10.1007/s00198-011-1804-x

[14] Silverberg, S.J., Shane, E., de la Cruz, L., et al. (1989) Skeletal Disease in Primary Hyperparathyroidism. Journal of Bone and Mineral Research, 4, 283-291. http://dx.doi.org/10.1002/jbmr.5650040302

[15] Rossini, M., Gatti, D., Isaia, G., et al. (2001) Effects of Oral Alendronate in Elderly Patients with Osteoporosis and Mild Primary Hyperparathyroidism. Journal of Bone and Mineral Research, 16, 113-119. http://dx.doi.org/10.1359/jbmr.2001.16.1.113

[16] Khan, A.A., Bilezikian, J.P., Kung, A.W., et al. (2004) Alendronate in Primary Hyperparathyroidism: A Double-Blind, Randomized, Placebo-Controlled Trial. Journal of Clinical Endocrinology \& Metabolism, 89, 3319-3325. http://dx.doi.org/10.1210/jc.2003-030908

[17] Grey, A.B., Stapleton, J.P., Evans, M.C., et al. (1996) Effect of Hormone Replacement Therapy on Bone Mineral Density in Post-Menopausal Women with Mild Primary Hyperparathyroidism. A Randomized, Controlled Trial. Annals of Internal Medicine, 125, 360-368. http://dx.doi.org/10.7326/0003-4819-125-5-199609010-00002

[18] Selby, P.L. and Peacock, M. (1986) Ethinylestradiol and Norethindrone in the Treatment of Primary Hyperparathyroidism in Postmenopausal Women. New England Journal of Medicine, 314, 1481-1485. http://dx.doi.org/10.1056/NEJM198606053142304

[19] Khosla, S. and Melton III, J. (2002) Fracture Risk in Primary Hyperparathyroidism. Journal of Bone and Mineral Research, 17, N103-N107.

[20] Cranney, A., Guyatt, G. and Griffith, L. (2002) Osteoporosis Methodology Group and the Osteoporosis Research Advisory Group. Meta-Analyses of Therapies for Postmenopausal Osteoporosis. IX: Summary of Meta-Analyses of Therapies for Postmenopausal Osteoporosis. Endocrine Reviews, 23, 570-578. http://dx.doi.org/10.1210/er.2001-9002

[21] Holvik, K., Ahmed, L.A., Forsmo, S., et al. (2013) Low Serum Levels of 25-Hydroxyvitamin D Predict Hip Fracture in the Elderly: A NOREPOS Study. Journal of Clinical Endocrinology \& Metabolism, 98, 3341-3350. http://dx.doi.org/10.1210/jc.2013-1468

[22] Heike, A., Bischoff-Ferrari, P.H., Walter, C., et al. (2012) A Pooled Analysis of Vitamin D Dose, Requirements for Fracture Prevention. New England Journal of Medicine, 367, 40-49. http://dx.doi.org/10.1056/NEJMoa1109617

[23] Moosgaard, B., Christensen, S.E., Vestergaard, P., et al. (2008) Vitamin D Metabolites and Skeletal Consequences in Primary Hyperparathyroidism. Clinical Endocrinology, 68, 707-715.

[24] Grey, A., Lucas, J., Horne, A., et al. (2005) Vitamin D Repletion in Patients with Primary Hyperparathyroidism and Coexistent Vitamin D Insufficiency. Journal of Clinical Endocrinology and Metabolism, 90, 2122-2126. http://dx.doi.org/10.1210/jc.2004-1772

[25] Berenson, J.R. (2002) Treatment of Hypercalcemia of Malignancy with Bisphosphonates. Seminars in Oncology, 29, 12. http://dx.doi.org/10.1053/sonc.2002.37417

[26] Bilezikian, J.P. (1993) Clinical Review 51: Management of Hypercalcemia. Journal of Clinical Endocrinology \& Metabolism, 77, 1445.

[27] Khanna, A. (2006) Acquirednephrogenic Diabetes Insipidus. Seminars in Nephrology, 26, 244-248. http://dx.doi.org/10.1016/j.semnephrol.2006.03.004

[28] Yu, N., Leese, G.P., Smith, D., et al. (2011) The Natural History of Treated and Untreated Primary Hyperparathyroidism: The Para-Thyroid Epidemiology and Audit Research Study. Quarterly Journal of Medicine, 104, 513-521. http://dx.doi.org/10.1093/qjmed/hcq261 
Scientific Research Publishing (SCIRP) is one of the largest Open Access journal publishers. It is currently publishing more than 200 open access, online, peer-reviewed journals covering a wide range of academic disciplines. SCIRP serves the worldwide academic communities and contributes to the progress and application of science with its publication.

Other selected journals from SCIRP are listed as below. Submit your manuscript to us via either submit@scirp.org or Online Submission Portal.
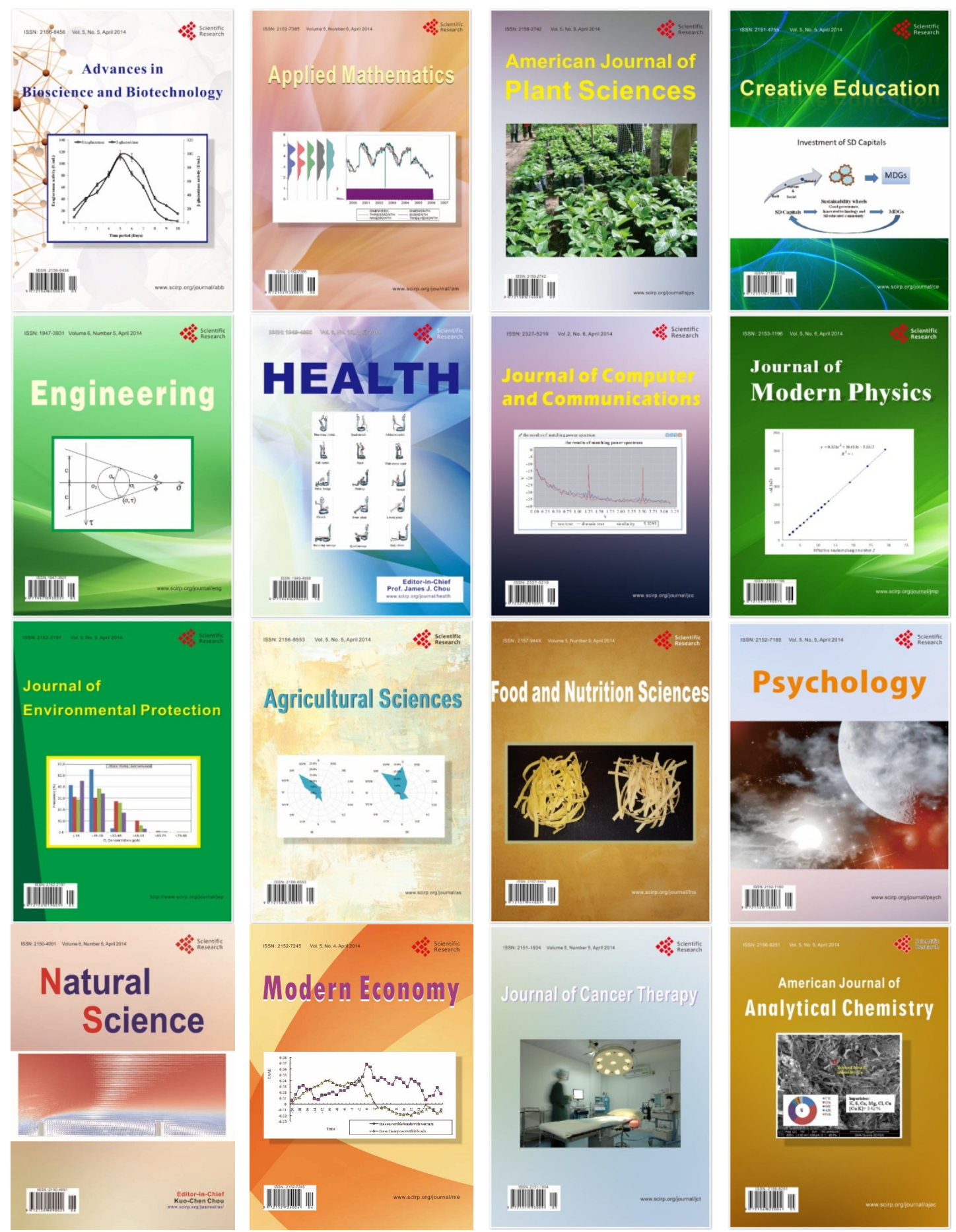